\title{
Subjective Perspectives and Direction Expressions in Modern Chinese
}

\author{
Chun Li \\ School of Liberal Arts \\ Nanjing University \\ Nanjing, China \\ Lothlorien513@gmail.com
}

\begin{abstract}
Modern Chinese expresses the spatial relationship by a variety of means. Spatial perspectives profoundly influence the grammatical and semantic structures, which is especially embodied in the effect of the subjective perspective on direction expression. There are two reasons for this. One is that the perspective embodies the speaker's subjective construal ways to a large extent, which leads to the subjectivity of language's perspective, the other is that as one of the most basic cognitive domains, spatial domain takes direction expression as its important part in the language level.
\end{abstract}

Keywords- Spatial perspective; direction expressions; modern Chinese

\section{INTRODUCTION}

Modern Chinese use abundant means to express spatial relationships, such as vocabulary, syntax, and discourse. Vocabulary means include direction words, location nouns, prepositions, deixis, and adjectives. Syntax means consist of phrases like "zai fangjian li" (in the room), and sentence forms like "qiang shang tiezhe yifu hua" (there is a picture on the wall). Spatial relationships are also connected with discourse structures. For a perspective system, the position and movement pattern of an observation point can be reflected in discourse level. For example, in the sentence group "ni jixu wang qian zou, qianmian you guai, jiu kanjian yiyuan le" (Go ahead and turn right. Then you will see the hospital).

Subjectivity refers to such a language characteristic that the speaker always wants to convey their standpoint, attitude and emotion while speaking. [1]Structural linguistics consider that the function of language as objective description instead of subjective construal, whereas cognitive linguistics indicate that language are both objective and subjective. [2] Subjectivity generally exists in languages, considering that speakers are able to use phoneme, intonation, vocabulary, syntax, discourse, expression language and even body language to express their self existence. For example, the space deixis "zhe"(this), personal pronoun "ni"(you), directional verb "lai"(come), direction word "zuo"(left) and time noun "jintian"(today) all indicate that speakers organize the utterance structure from ego's standpoint to express time, space and interpersonal relationships.

People construe and describe physical space from their own perspective. Linguistic space is the reflection of mental space, while the latter re-reflects physical space. In that sense, semantic and syntactic structures are not necessarily with the reality. Subjective perspective in spatial domain forms when people cognize and depict space in their personal ways.

\section{II. "NOUN+ DIRECTION" WORD PHRASES AND ATTENTION SCOPE}

Although the combination relation of nouns and direction words is a syntactic matter, the selection of direction words is based on how people recognize geometric properties of object. Liu Ningsheng maintained that it is a process of filtration to transform geometric properties of object into expression. In the process of cognition, when some properties of object are magnified, others are minimized or even filtered.[3] In other words, the more obvious the properties are, the easier they are perceived by people. The law above is prominently reflected in Chinese in terms of combination of nouns and direction words. For instance, when people say "dimian shang"(on the ground), they imagine the earth as flat instead of sphere; when they say "chouti li "(in the drawer), they recognize that the drawer has a inner space.

The essential difference between bookcase and bookshelf is whether it has doors. The reason why we say "shu zai shuchu li"( The book is in the bookcase) instead of "shu zai shuchu shang"(The book is on the bookcase) is we regard bookcase as a case or a box. As for bookshelf, we see it as composed of several flats, so we say "shu zai shujia shang"(The book is on the bookshelf) instead of "shu zai shujia li"(The book is in the bookshelf).

The equivalent of "zai xuexiao li" in Chinese is "at the school" or "on the campus" in English. Chinese people regard school as a space separated from outside world, while English people regard it as a spot or a plane. It is said that the combination of nouns and direction words in different languages varies with attention scope, which is based on certain criterion for the degree of significance of geometric properties. it proves that there is a relationship between selection of perspective and spatial knowledge of people.

\section{THE DEGREES OF FREEDOM OF THE "NOUN + DIRECTION WORD" COMBINATION FROM THE PERSPECTIVE OF SUBJECTIVITY}

Influenced by the object attribute salience and the national psychological commonality, native language learners often construe space object similarly. A noun is a conceptual reference to space objects in a language, so the "noun + direction word" combination also has a tendency. For example, 
in Chinese, there are such expressions as "huoche shang"( on the train), "chexiang li"(in the carriage), "xiaoyuan zhong"(in the campus)", instead of "huoche li"(on the train), "chexiang shang"(in the carriage), "xiaoyuan shang"(in the campus).

We have a common thinking tendency in the construal of things, but this does not mean that we will be restricted by the stipulation all the time. On the contrary, under certain conditions, everyone can make a subjective description of things based on his or her own perspective, which reflects the dialectics of absolute and relative. In English, the same noun can match different direction word, which reflects the degrees of freedom of the perspective subjectivity of cognition subject and phrases' collocations.

For example, "in the trees" and "on the tree" are both expressed as "shu shang" (tree up/on) in Chinese, emphasizing only being higher than the spatial location of the observation point and undistinguishing the space' two-dimensional or three-dimensional in the subjective construal. Similarly, "On the windows" and "in the window" are expressed as "Chuang Shang"(window up/on) in Chinese, which does not distinguish the space' two-dimensional or three-dimensional in the subjective construal. On the grass" and "in the grass" respectively corresponds to "caodi shang" (meadow on) and "caocong li" (medow in), which embody the meadow both in English and in Chinese can be subjectively construed as a twodimensional or three-dimensional space. However, except that different direction words are used in Chinese as in English, Chinese can realize the subjective perspective by distinguishing the nuance of the double syllable words containing the same morpheme. This shows that the same things' subjective degree and freedom degree of syntactic combination are different to people from different regions or nations. The perspective subjectivity exists not only in the phrase level, also in the sentence level. For example[4]:

(1)The hill falls gently to the Bank of the river.

(2)The hill rises gently from the bank of the river.

As can be seen, the verb fall in sentence embodies the speaker's "top-down" perspective, and the verb rise in sentence 10 embodies the speaker's "down-up" perspective. Nevertheless, the hills and the river bank are actually in the static state in the same space. Therefore, the two expression forms reflect the speaker's different perspective experience to the same state of the things.

\section{THE SPECIAL USAGE OF THE DIRECTION WORDS “SHANG”(UP) AND “XIA"(DOWN) FROM THE DIRECTION OF OBSERVATION}

The direction word "shang"(up) can be replaced with its antonym "xia"(under) in some direction phrases without changing their meanings. For example, "di shang" is equal to "di shang" in mandarin and Yangzhou dialect in China , both means "on the ground".[5] We believe that this is the result of the speaker's choosing different reference framework. When "di shang"(ground up) is used, the speaker's intrinsic reference frame works, which has nothing to do with the observer. "shang"(up) is the inner azimuth the ground itself has, "di shang" is a projection of mirror image of space relationship in the physical space. Similarly, when "di xia"(ground down) is used, the speaker's relative reference frame works, which identifies the position relationship between objects from egocentric perspective. Therefore, "underground" implies the gaze direction of the speaker. In other words, the eyes above the ground, the speaker observes things on the ground with his gaze down.

Here are another example, "tianhuaban shang you yizhi cangying" is equal to "tianhua banxia you yizhi cangying", which means "there is a fly on the ceiling".In physical space, the fly is indeed on the lower surface of the ceiling, but "tianhuaban shang"(ceiling on) is actually expressed in Chinese, which is also because the speaker construes the space scene in the relative reference frame. "tianhuaban shang"(ceiling on) implies the gaze direction of the speaker. In other words, the speaker has to observe the things under the ceiling with his gaze up, because his eyes are below the ceiling.

Both "di shang"(ground up) and "tianhuaban xia"(ceiling down) reflect intrinsic reference frame, but "di shang"(ground up) can be said while "tianhuaban shang"(ceiling up) cannot. Why? We hypothesize that one of the reasons is the asymmetry of "up" and "down", which is embodied in the early or late time of acquisition and in the frequency of its being used.

"shang"(up) and "xia"(down) are both high frequency words, but have different frequency in the actual use. The statistics of about 1061 ten thousand words time Chinese natural corpus offered by Xiamen University shows that "shang"(up) appears 49841 times in all in the corpus and its word frequency is about 4696 per million, and that "xia"(down) appears 31800 times in all in the corpus and its word frequency is about 2997 per million. The ratio is nearly the same in English. According to "the British National Corpus" (website: http://natcorp.ox.ac.uk) statistics, the word frequency of "up, on, above" are all higher than that of down, under and below. All these show that "shang"(up/on/above) are more universal than "xia"(down/under/below).[6]

The acquisition of "up/on/above" is generally earlier than that of "down/under/below" when children of different nationalities learn spatial words. The acquisition order of spatial words in the five languages including is shown as in Table I [7]:

TABLE I THE ACQUISITION ORDER OF SPATIAL WORDS IN THE FIVE LANGUAGES

\begin{tabular}{|c|c|c|c|c|c|}
\hline English & Italia & Serbia-Croatia & Turkish & Chinese & sequence \\
\hline in & in & on & in & in & \\
\hline on & on & in & on & on & \\
\hline under & under & beside & under & under & \\
\hline beside & beside & under & beside & & \\
\hline between & between & back & back $_{f}$ & back & \\
\hline front & back & between & front $_{f}$ & between & \\
\hline back & front & front $_{f}$ & between & front & \\
\hline back & back & back & back & outside & \\
\hline front & front & front & front & beside & \\
\hline
\end{tabular}


Slobin considers that the acquisition of language markers by children can be categorized four stages: (a) the unmarked; (b) the use of suitable markers with limited language knowledge; (c) the over generalization of markers; (d) the complete acquisition of the language.[8] In other words, the acquisition of unmarked forms in language is earlier than the acquisition of the marked forms. Based on the semantic scope and syntactic distribution of "up" and "down", Shen Jiaxuan argues that "up" as an unmarked spatial preposition, is acquired earlier than "down" as a marker one.[9]

In a language, a pair of opposite language elements does not necessarily have the same amount. One element (unmarked) is often more basic, more natural, more common than its opposite (marked). Moreover, some psychological experiments show that it takes less time for children to process unmarked items as "up, front, in, come" than marked items as "down, back, outside, go". [10]

We speculate that "up" may have advantages over "down" when competing for the syntactic position of the same semantic meaning. Therefore, "di shang"(ground up/on) is used more frequently than "di xia"(ground down/below) to convey the meaning of "on the surface of the ground". Another reason for low frequency of "di xia" might be its ambiguity, namely "di xia" usually means below the surface, such as "zhe pian shamo de di xia yuancang zhe fengfu de kuangcang"(abundant supply of mineral resources are contained underground the desert). "Tianhuaban shang"(ceiling on) is frequently used to convey the meaning of " the lower surface of the ceiling", and "tianhuaban xia"(ceiling under) is almost never used. Similarly, another reason might be the ambiguity of "tianhuaban xia", namely, it can denote the space region below the ceiling, such as "shanqu de haizi men zai yaoyaoyuzui de tianhuaban xia xuexi"'(children in mountainous area are studying under the dilapidated ceiling).

\section{AMBIGUITIES OF DIRECTION WORDS AT THE} HORIZONTAL DIMENSION FROM THE POSITION OF OBSERVATION AND REFERENCE STRATEGIES OF SPATIAL DECISION IN CHINESE

There are two kinds of spatial expressions: one is the expression of absolute space, which does not need reference objects, such as "in Beijing", "in the school", and another is the expression of relative space, which need reference objects, such as "in front of the car", "on the left of the TV". The expression of relative space usually takes advantage of the three dimensions of space: 1. The vertical dimension, i.e. the upper/lower dimension; 2.The longitudinal horizontal dimension, namely front/back dimension; 3. The horizontal dimension, namely left/right dimension.

The expression of relative space of horizontal dimension often creates ambiguity, as is shown in sentence 3 in English. "in front of" can denote two positions of a and b.(see Figure 1)

(3) There is a cat in front of the car.

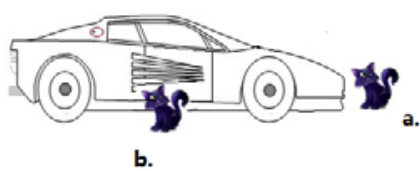

FIGURE I IN FRONT OF THE CAR"

(4)Qiche qianmian you yizhi mao.(There is a cat in front of the car)

Guo Rui investigated 97 subjects on sentence 4.The results show that the similar expressions in Chinese can denote four kinds of positions (see Figure 2).[11]

$100 \%$ of subjects choose position (a). $50 \%$ of subjects choose position(b). $18 \%$ of subjects choose (c) and nobody chooses (d). Ambiguity is mainly due to different reference strategies, which refers to how relative spatial expression takes advantage of reference objects' direction characteristics. (a) and (d) take advantage of intrinsic direction characteristics of reference objects, in (b) the speaker projects his/her mirror of direction characteristics on the reference object, and in (c) the speaker projects his/her direction characteristics clockwise on the reference object. The differences between (a) and (d) lies in whether the direction domain is a part of the reference object. In English, without the definite article "the", the direction domain is not a part of the reference object. If it is a part of the reference object, "the" has to be used. For example, "in front of the house" denotes the front outside the house, "in the front of the house" denotes the front in the house.

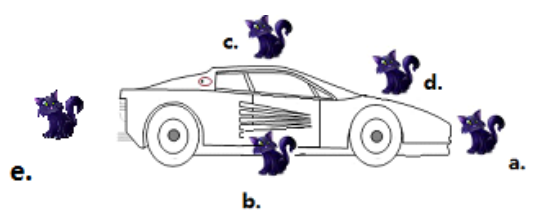

FIGURE II

FOUR POSITIONS DENOTED BY "QICHE QIANMIAN YOU YIZHI MAO”(THERE IS A CAT IN FRONT OF THE CAR)

As for the ambiguity in sentence 3, Clark argues that "front/back" in English has two denotations: 1. The front/back in the internal position of the reference object (position a); 2 . The front/back from the perspective of the speaker (position $b$ ). [12]As for the direction expression in Chinese on the horizontal dimension, Fang Jingming believes that in the use of "left/right" in Chinese, the perspective of subject and object have to be discriminated. The former equals Clark's "the direction from the perspective of the speaker", and the latter equals Clark's “internal position of the reference object".[13]

However, previous studies cannot explain why all the four positions in Chinese can be accepted and why there are different possibilities of being accepted for the three positions of which direction domain is not a reference object: position $\mathrm{a}>$ position $\mathrm{b}>$ position $\mathrm{c}>$ position $\mathrm{e}$.

We believe that the position of observation can account for the ambiguity in sentence 4 . First, we have to clarify a few presets: 1. Four basic relative directions on horizontal dimension is front, back, left and right, which means that there are 4 basic positions of observation when a cat is located with 
the car as a reference object. 2 . The prototypical meaning of "front" in the spatial domain is the front the observer is actually facing, and its analogical meaning is the front the objects except the observer himself or herself are facing (in the case that the facing direction of objects can be discriminated. The premise is the object has internal features discriminating the front from the back, such as the front being the direction of the side with a door in a house). 3. The analogical meaning of "front" is deduced from its prototypical meaning. Therefore, when focus is located in front of an object as the reference object, the direction from the perspective of the observer is tended to be viewed as the direction of the object, which is said to be construal operation of "subjectivity of the objects". Accordingly, "observer $\rightarrow$ reference object $\rightarrow$ focus object" being a linear sequence, the reference object will be construed as being in front of the reference object.

Now, it is assumed that four observers are observing the relationship between the car and the cat separately from four basic observing positions. If the factor of object itself is excluded, each observer will take the direction of his own observing position as the direction of the car. Therefore, position a, b, c, and e, theoretically, all can be where the cat is located in front of the car. However, the internal characteristics of the car (vehicle with front and back; the front is the engine cover, and the back is the tail cover) itself can distinguish between front and back. Position a is near the front of the car, and position $b$ is near the back of the car. As inherent attributes, the internal characteristics of the car decide the "front" and the "back", which is objective and fixed, while the "front" and the "back" construed by subjectivity of the objects is subjective and relative. The former's directional characteristics in a strong position, called "strong condition", will be the first choice of the observer, while that of the latter in a weak position, called "weak condition", will not be the observer's first choice. Therefore, on the condition that the conception of "front" and "back" of the car itself is not violated, the observer will judge "front/back" from his/her own observation direction. Position judged as being "front" before will never be judged as being "back" once again, and position judged as being "back" before will never be judged as being "front".

According to this rule, position e is first excluded, because it is the "strong condition" that judges it as the "back", and it is the "week condition" that judges it as the "front". "Strong condition" is preferred to "weak condition". Position a has the highest degree of acceptance in that both the strong condition and weak condition judge it as the "front", which has the strongest motivation. Strong condition cannot judge position $b$ and position c as "back", and weak condition judges position $\mathrm{b}$ and position c as "front". Why the degree of acceptance of position $b$ is higher than that of position $c$ ? We believe that the reason is that the subject acquires the spatial relationship scene of "car - cat" from the right side of the car. From the car's right side, the subject observes position $\mathrm{b}$ without shelter and position c with shelter. However, the prototypical meaning of "front" implies "visible in the area the observer faces", and that of "back" implies "invisible in the opposition area of the observer's direction. Hence, the subject tends to judge position b without shelter as "front", and position c with shelter as "back". Strengthened by the additional condition, position $b$ has higher degree of acceptance than position c.

The analysis of the ambiguity of direction expressions on horizontal dimension gives us insight into reference strategies of spatial decision in Chinese. Spatial decision is related to the inherent direction characteristics of objects, the observation position the observer subjectively chooses, and the observation position in which the observer acquires the space scene. It is a variety of subjective construal that leads to ambiguity of direction expression on horizontal dimension. Nevertheless, there are different degrees of acceptance to the direction relationship embodied in all the meanings conveyed in ambiguous sentences, which is caused by simultaneous constraints of a series of conditions on direction decision.

\section{ACKNOWLEDGEMENTS}

The study is funded by 2014 Annual Innovative Research Program for Postgraduates in Jiangsu Province in China: The study of second languages' word order error based on corpus (KYZZ_0012).

\section{REFERENCES}

[1] Lyons, J. Linguistics Semantics:An Introduction. Cambridge: Cambridge University Press, 1995

[2] Shen Jiaxuan. A survey of studies on subjectivity and subjectivisation. Foreign Language Teaching and Research, 2001.

[3] Liu Ningsheng How Chinese express spatial relationships of objects. Studies of The Chinese Language, 1994.

[4] Langacker,R.W, Foundations of Cognitive Grammer. Stanford: Stanford University Press, 1987.

[5] Chu Zexiang, Xiao Renfei, The Reference System of “ Dixia” and Its Matching Restriction. Language Teaching and Linguistic Studies, 2010.

[6] Bai Lifang, The Symmetry and A symmetry in the Semantic Structure of "N. +shang/xia " . Language Teaching and Linguistic Studies, 2006

[7] Jia Hongxia, the development law of spatial category of Mandarin children. PhD dissertation of the Graduate School of Chinese Academy of Social Sciences, 2009.

[8] Slobin, D, The role of language in language acquisition. Invited address to the 50th Annual Meeting of the Eastern Psychological As sociation, Philadelphia. Unpublished ms., Berkeley, CA: University of California, 1979.

[9] Shen Jiaxuan, Asymmetry and the Marked Theory. Nanchang:Jiangxi Education Publishing House, 1999.

[10] Richards, Jack, John Platt and Heidi Weber, Longman Dictionary of Ap-plied Linguistics, London: Longman, 1985

[11] Guo Rui, Spatial reference theory and Chinese spatial expression reference strategy.Included in Contemporary linguistics theory and Chinese Research. Beijing: The Commercial Press, 2008.

[12] Clark, E. V., Nonlinguistic strategies and the acquisition of word meanings. Cognition 2, 1973.

[13] Fang Jingmin, Subjective and Objective View in "Zuo/you" Direction reference in Chinese. Language Teaching and Linguistic Studies,1987. 